\title{
Osteoporosis en pacientes con artritis reumatoide que reciben corticoides a dosis bajas
}

Osteoporosis in patients with rheumatoid arthritis receiving low-dose of corticosteroids

Guevara Serrano Karen Dayana'1, Guzhñay Bernal Silvia Maribel $^{1}$

VOLUMEN 38 | N 3 | DICIEMBRE 2020

FECHA DE RECEPCIÓN: 04/01/2021 FECHA APROBACIÓN: 24/02/2021 FECHA DE PUBLICACIÓN: 06/04/2021

1. Médica en libre ejercicio. Cuenca - Ecuador

\section{Artículo $\quad$ Original Original Article}

DOI: https://doi.org/10.18537/RFCM.38.03.03

Correspondencia:

karen.guevaras@ucuenca.edu.ec

Dirección:

Paseo 3 de Noviembre y los

Cerezos

Código Postal:

010219

Teléfono:

0984147110

Cuenca - Ecuador

\section{RESUMEN}

La Osteoporosis (OP) es una comorbilidad frecuente en pacientes con Artritis Reumatoide (AR) que toman dosis bajas de glucocorticoides (GCs) (<7.5mg), lo que ocasiona mala absorción del calcio, disminución de la masa ósea y riesgo de fractura.

Objetivo: establecer la frecuencia de OP y Densidad Mineral Ósea (DMO) baja en pacientes con AR que toman dosis bajas de GCs del Hospital Vicente Corral Moscoso (HVCM).

Metodología: estudio descriptivo de corte transversal, realizado en pacientes con AR atendidos en consulta externa del HVCM, que reciben además de la medicación convencional drogas antirreumáticas modificadoras de la enfermedad (DMARDs) y dosis bajas de GCs. La muestra fue calculada en base a una frecuencia del 30\% de OP. Para el diagnóstico de OP se utilizó densitometría axial (DXA) con los criterios OMS. La información se procesó en SPSS versión 15.

Resultados: el estudio se realizó con 161 pacientes con AR que tomaban dosis bajas de GCs, el 96.3\% fue del género femenino, el grupo etario de 40 a 64 años representó el $60.2 \%$, la mayoría se desempeña en quehaceres domésticos con un 78.9\%. La DMO en columna lumbar fue baja con $39.1 \%$ y OP $37.9 \%$; el DMO en cuello femoral fue $39.8 \%$ y OP $17.4 \%$.

Conclusiones: se encontró frecuencia de OP y DMO disminuida en pacientes con AR que toman GCs en dosis bajas; siendo, más frecuente la OP y DMO disminuida en mayores a 64 años con AR y que toman GCs durante más de diez años.

Palabras clave: artritis reumatoide, osteoporosis, glucocorticoides, factores socioeconómicos, factores de riesgo. 


\section{ABSTRACT}

Osteoporosis (OP) is a frequent comorbidity in patients with rheumatoid arthritis (RA) who take low doses of glucocorticoids (GCs) $(<7.5 \mathrm{mg})$, which causes malabsorption of calcium, decreased bone mass and risk of fracture.

Objective: to establish the frequency of $\mathrm{OP}$ and low bone mineral density (BMD) in patients with RA who take low doses of GCs from the Vicente Corral Moscoso Hospital (HVCM).

Methodology: a descriptive, cross-sectional study was carried out in patients with RA who were attended in the HVCM outpatient clinic, who receive disease-modifying anti-rheumatic drugs (DMARDs) and low doses of GCs in addition to conventional medication. The sample was calculated based on a frequency of $30 \%$ of OP. For the diagnosis of OP, axial densitometry (DXA) was used with WHO criteria. The information was processed in SPSS version 15 .

Results: the study was carried out with 161 patients with RA who took low doses of GCs, $96.3 \%$ were female, the age group from 40 to 64 years represented $60.2 \%$, the majority made domestic chores 78.9\%. The BMD in the lumbar spine was low with $39.1 \%$ and OP $37.9 \%$; low BMD in the femoral neck $39.8 \%$ and OP $17.4 \%$.

Conclusions: frequency of OP and decreased BMD was found in RA patients taking low-dose GCs; being more frequent the OP and decreased BMD in people older than 64 years with RA and who take GCs for more than ten years.

Key words: rheumatoid arthritis, osteoporosis, glucocorticoids, socioeconomic factors, risk factors.

\section{INTRODUCCIÓN}

La OP se caracteriza principalmente por una DMO baja, con deterioro de su micro arquitectura ósea e incremento del riesgo de fracturas ${ }^{1}$; su prevalencia se ha incrementado a nivel mundial por envejecimiento de la población, consumo de GCs y modificaciones en el estilo de vida como el sedentarismo². Aproximadamente más de 200 millones de personas en el mundo padecen $\mathrm{OP}^{3}, 10$ millones de casos se reportan en Brasil ${ }^{4}$. Las fracturas son causa de morbimortalidad, en un estudio realizado en México se proyectó que 1 de cada 12 mujeres y 20 hombres mayores de 50 años, presentarán fractura de cadera ${ }^{5}$.

Los pacientes con AR pueden desarrollar OP ocasionada por diversos factores: interleucina-6 (IL-6), mediadores de RANK que activan los osteoclastos y fármacos como los GCs, que alteran la estructura ósea ${ }^{6}$. Algunos ensayos clínicos han demostrado la utilidad de GCs en el tratamiento de la AR, combinando con DMARDs, así como terapia puente; sin embargo, los GCs pueden disminuir la absorción del calcio en el tubo digestivo y acciones de la vitamina D. Por lo que dosis altas o bajas a largo tiempo, están asociados con riesgo de OP y otras comorbilidades. Un estudio realizado en Azuay, demostró que pacientes de sexo femenino con AR presentan OP de columna en el $34.6 \%$ y de cuello femoral el $30.85 \%$.

La densitometría axial de columna lumbar anteroposterior y de fémur proximal resulta ser el método más confiable, de acuerdo a la OMS, para medir la DMO y diagnosticar OP; a la vez es prueba necesaria para la predicción de futuras fracturas osteoporóticas e implementar la intervención terapéutica oportuna-10. El presente estudio establece la frecuencia de la DMO baja y OP a través del examen de DXA en 
pacientes con AR que toman dosis bajas de GCs $(<7.5 \mathrm{mg})$, información que permitirá realizar la prevención de fracturas por fragilidad.

\section{METODOLOGÍA}

Se realizó un estudio de tipo descriptivo y de corte transversal en el cual se consideró al total de pacientes con diagnóstico de AR que reciben atención en el servicio de reumatología del área de consulta externa por parte de los especialistas que laboran en el HVCM, en el período comprendido entre mayo del 2017 hasta abril del 2018.

Dentro de los criterios de inclusión en este estudio se tomaron en cuenta aquellos pacientes que completaron los criterios de diagnóstico de 1987 de ACR para AR 11,12, tener AR más de 2 años, estar en tratamiento con GCs a dosis bajas ( $<7.5 \mathrm{mg}$ ) con un mínimo de tres meses y firmar el consentimiento informado. Como criterios de exclusión los pacientes que estén cursando otra enfermedad que afecte la masa ósea, pacientes que se encuentren en tratamiento con dosis altas (20mg) de GCs y aquellos que no posean un estudio DXA.

Constituyeron el universo del estudio un total de 624 pacientes de consulta externa de reumatología del HVCM, de los cuales 320 toman dosis bajas de GCs $(<7.5 \mathrm{mg})$; aplicando un intervalo de confianza al 95\%, una prevalencia del $30 \%$ y una precisión del $5 \%$, se determinó que el tamaño de la muestra es de 161 pacientes.

El diagnóstico de la OP O DMO baja fue realizado con estudio de DXA, que mide la densidad de área en gramos por centímetro cuadrado $\left(\mathrm{g} / \mathrm{cm}^{2}\right)$, se obtiene el T-score en desvío estándar (DE) al comparar el DMO del paciente con la media de una población normal de adultos jóvenes del mismo sexo y raza. La medición más adecuada se realiza en columna lumbar y cadera derecha, sitios más propensos a la pérdida ósea y mayor riesgo de fractura. La interpretación siguió criterios de la OMS: normal DMO >-1 DE T-score; osteopenia DMO entre -1 DE y -2.5 DE T-score y OP DMO <-2.5 DE T-score ${ }^{13}$. Se utilizó equipo marca Hologic, serie 84741 del centro Medimagen en la ciudad de Cuenca.

Para el registro y tabulación de los datos se usó: Excel 2013, SPSS versión 15. La recolección de datos se coordinó con médicos reumatólogos que laboran en el área de consulta externa del HVCM para realizar la encuesta, así como la firma del consentimiento informado. Se utilizó formulario creado por los autores para completar la información necesaria en este estudio. El análisis estadístico fue bivariado de cada una de las variables utilizadas.

\section{RESULTADOS}

En la Tabla $\mathrm{N}^{\circ} 1$ se observa que el $60.2 \%$ corresponde a los adultos entre 40-64 años, seguido de un 33.6\% de adultos mayores de 65 años. Según el sexo predominan las mujeres con el 96.3\%. De acuerdo al lugar de residencia se observa que un $89.4 \%$ reside en la provincia del Azuay y en ocupación los datos indican que el $78.9 \%$ se dedica a los quehaceres domésticos. 


\section{Tabla $\mathrm{N}^{\circ} 1$}

Características sociodemográficas de 161 pacientes con Artritis Reumatoide que toman corticoides a dosis bajas del HVCM

\begin{tabular}{lrr}
\hline & $\mathbf{n}$ & $\%$ \\
\hline Edad & & \\
$\quad$ Adulto joven 20-39 años & 10 & 6.2 \\
$\quad$ Adulto 40-64 años & 97 & 60.2 \\
$\quad$ Adulto mayor +65 años & 54 & 33.6 \\
Sexo & & \\
$\quad$ Hombre & 6 & 3.7 \\
$\quad$ Mujer & 155 & 96.3 \\
Residencia & 144 & \\
$\quad$ Azuay & 17 & 89.4 \\
Otra provincia & & 10.6 \\
Ocupación & 1 & 0.6 \\
Sin trabajo & 23 & 14.3 \\
Con trabajo & 10 & 6.2 \\
Jubilado/a & 127 & 78.9 \\
Quehaceres domésticos & & \\
\hline
\end{tabular}

Dentro de los datos más relevantes a destacar en las diferentes tablas cruzadas se puede observar que en el grupo etario mayores de 65 años prevalece en el 63\% la OP frente a los otros grupos (Tabla N²).

\section{Tabla $\mathrm{N}^{\circ} 2$}

Distribución de 161 pacientes con Artritis Reumatoide de consulta externa del HVCM según su edad y Osteoporosis

\begin{tabular}{cccc}
\hline $\begin{array}{c}\text { Resultado de } \\
\text { Osteoporosis }\end{array}$ & Adulto joven 20-39 años & Adulto 40-64 años & Adulto mayor $>\mathbf{6 5}$ \\
\hline Sí & $2(20 \%)$ & $36(37.1 \%)$ & $34(63 \%)$ \\
No & $8(80 \%)$ & $61(62.9 \%)$ & $20(37 \%)$ \\
\hline
\end{tabular}

En la tabla $N^{\circ} 3$ se describe la relación entre el tiempo en el que los pacientes han sido diagnosticados de AR y el examen DXA para determinar OP, dando como resultado que en personas que padecen AR entre 5 a 10 años el 35.8\% presentan OP y aquellos pacientes con más de 10 años el 60\% tiene OP.

\section{Tabla $N^{\circ} 3$}

Pacientes con Artritis Reumatoide que padecen Osteoporosis según tiempo de evolución

\begin{tabular}{cccc}
\hline Resultado de & \multicolumn{3}{c}{ Tiempo que padece Artritis Reumatoide } \\
Osteoporosis & Menor de 5 años & 5 a 10 años & Mayor de 10 años \\
\hline Sí & $2(8.7 \%)$ & $19(35.8 \%)$ & $51(60 \%)$ \\
No & $21(91.3 \%)$ & $34(64.2 \%)$ & $34(40 \%)$ \\
\hline
\end{tabular}


En la tabla $\mathrm{N}^{\circ} 4$ se muestra la relación entre el tiempo que toman los pacientes dosis bajas de GCs, a quienes se realizó DXA en columna vertebral, en la interpretación de T-score el 46.6\% presentó osteopenia en el grupo de 5 a 10 años con AR; en tanto que la OP se observa en porcentaje del 55.2\% en personas que han tomado dosis bajas de GCs por más de 10 años.

\section{Tabla $\mathrm{N}^{\circ} 4$}

Densitometría ósea en cuello femoral de pacientes con Artritis Reumatoide con dosis bajas de corticoides según años de enfermedad

\begin{tabular}{lccc}
\hline \begin{tabular}{c} 
Interpretación de T score en \\
\multicolumn{1}{c}{ columna vertebral }
\end{tabular} & \multicolumn{3}{c}{ Tiempo de toma de corticoides } \\
\hline Normal & $10(27.8 \%)$ & $15(25.9 \%)$ & $12(17.9 \%)$ \\
Osteopenia & $18(50 \%)$ & $27(46.6 \%)$ & $18(26.9 \%)$ \\
Osteoporosis & $8(22.2 \%)$ & $16(27.6 \%)$ & $37(55.2 \%)$ \\
\hline
\end{tabular}

Por otra parte, se observa que en relación a T-score de columna vertebral el 50.9\% de pacientes que tienen AR entre 5 y 10 años presentan osteopenia; en tanto, que aquellos con AR más de 10 años en el $48.2 \%$ evidencian OP (Tabla $N^{\circ} 5$ ).

\section{Tabla $N^{\circ} 5$}

Densitometría ósea en columna vertebral de pacientes con Artritis Reumatoide con dosis bajas de corticoides según años de enfermedad

\begin{tabular}{lccc}
\hline \begin{tabular}{l} 
Interpretación de T score en \\
\multicolumn{1}{c}{ columna vertebral }
\end{tabular} & \multicolumn{3}{c}{ Tiempo que padece Artritis Reumatoide } \\
& Menor de 5 años & 5 a 10 años & Mayor de 10 años \\
\hline Normal & $14(60.9 \%)$ & $8(15.1 \%)$ & $15(17.6 \%)$ \\
Osteopenia & $7(30.4 \%)$ & $27(50.9 \%)$ & $29(34.1 \%)$ \\
Osteoporosis & $2(8.7 \%)$ & $18(34 \%)$ & $41(48.2 \%)$ \\
\hline
\end{tabular}

\section{DISCUSIÓN}

Los pacientes con AR, enfermedad inflamatoria autoinmune crónica, requieren de tratamientos que incluyen DMARDs, biológicos y un porcentaje importante GCs; sin embargo, el tratamiento con los GCs, inducen la disminución de la masa ósea ${ }^{14} \mathrm{e}$ incrementa el riesgo de fractura. La pérdida de masa ósea en pacientes tratados con GCs puede variar de 3\% al 20\% en la DMO de 1 a 2 años, aumentando el riesgo de fracturas de vértebras o costillas, que están constituidos de hueso trabecular, a la vez que puede acrecentar las fracturas en caderas después de 5 a 10 años de tratamiento ${ }^{15}$.
Un estudio de cohorte en el Reino Unido demostró que la AR afecta al $68 \%$ de mujeres ${ }^{16}$. En la población de Saraguro, provincia de Loja, a través del COPCORD de enfermedades reumáticas, se observó prevalencia de AR con el $1.3 \%$ predominando las mujeres con $91 \%{ }^{17}$. En el presente estudio el $96.3 \%$ de pacientes con AR que toman GCs en dosis bajas fueron mujeres, lo que podría relacionarse con una mayor frecuencia de OP, establecida especialmente en pacientes con niveles bajos de estrógenos ${ }^{18}$, que ocurre por diversas causas, entre ellas la menopausia ${ }^{19}$.

Al revisar los aspectos sociodemográficos de este estudio, el $60.2 \%$ pertenece al grupo etario 
de 40 a 64 años de edad, similar a lo reportado porBullock et $\mathrm{a}^{20}{ }^{20}$ en donde la edad comprendida entre los 35 a 60 años es un factor de riesgo para el desarrollo de AR. Importante reconocer que de las comorbilidades el $62.1 \%$ presentan diabetes mellitus superior a lo reportado en pacientes mexicanos con AR (12.4\%); y, 16.8\% HTA, inferior a lo reportado en el mismo estudio en México $(29.8 \%)^{21}$.

El uso de fármacos como los GCs a largo plazo puede alterar el metabolismo óseo y mineral, incrementando la probabilidad de OP y riesgo de fractura ${ }^{22}$. En el presente estudio se observa que los pacientes con AR que tienen más de 10 años tomando GCs muestran mayor riesgo de OP con el $55.2 \%$, superior a lo reportado en un estudio en China que mostró el $41.6 \%{ }^{23}$.

Los resultados de este estudio demostraron que los pacientes con diagnóstico de AR que toman dosis bajas de GCs presentaron en columna vertebral osteopenia $39.1 \%$ y OP del $37.9 \%$ siendo este último mayor a lo reportado en población africana con el 14\%24; aunque, resulta ser menor a lo hallado en China en donde se evidencia $41.6 \%{ }^{23}$. Por otra parte, en cuello femoral el presente estudio nos da un reporte de osteopenia del $39.8 \%$ y OP del $17.4 \%$, menor a lo indicado por Santillán ${ }^{7}$ de $28.29 \%$ y mayor a Mikuls ${ }^{24}$ con el 6\%. Observándose que con el uso de GCs compromete más la columna lumbar.

Algunos estudios han señalado que una dosis de 5mg a 9mg/día de GCs, afecta especialmente el hueso trabecular; sin embargo, para otros autores, la disminución de la masa ósea no dependería de la dosis sino del tiempo acumulado. Lo que conlleva al riesgo de fractura por la posibilidad que los esteroides alteren la calidad del hueso ${ }^{25}$. En el presente estudio se consideró dosis de GCs $<7.5 \mathrm{mg}$.

\section{CONCLUSIÓN}

En el presente estudio se observó una alta frecuencia de OP y DMO disminuida en pacientes con AR que toman GCs en dosis bajas; siendo, más frecuente en pacientes mayores a 64 años con AR y que ingieren GCs como tratamiento de la enfermedad por más de diez años.

\section{ABREVIATURAS}

AR: artritis reumatoide

DE: desvío estándar

DMARDs: drogas antirreumáticas modificadoras de la enfermedad.

DXA: densitometría axial

DMO: densidad mineral ósea

GCs: glucocorticoides

HVCM: Hospital Vicente Corral Moscoso

OP: osteoporosis

\section{ASPECTOS BIOÉTICOS}

El protocolo fue entregado a la Comisión de Proyectos de Investigación, donde fue aprobado por el Comité de Bioética y por el Consejo Directivo de la Facultad de Ciencias Médicas de la Universidad de Cuenca. El presente trabajo se realizó bajo el consentimiento informado del paciente con total confidencialidad de los datos personales.

\section{INFORMACIÓN DE LAS AUTORAS}

Guevara Serrano Karen Dayana. Médica. Libre ejercicio. Cuenca-Azuay-Ecuador.

e-mail: karen.guevaras@ucuenca.edu.ec

ORCID:https://orcid.org/0000-0003-3023-2841

Guzhñay Bernal Silvia Maribel. Médica. Libre ejercicio. Cuenca-Azuay-Ecuador.

e-mail: silvismagu@gmail.com

ORCID:https://orcid.org/0000-0002-4555-976X 


\section{CONTRIBUCIÓN DE LOS AUTORES}

Las autoras declaran haber contribuido de manera similar en la elaboración del presente manuscrito.

\section{CONFLICTO DE INTERESES}

No existe conflicto de intereses.

\section{FUENTES DE FINANCIAMIENTO}

Fondos propios.

\section{AGRADECIMIENTO}

A los pacientes y familiares que colaboraron en la presente investigación.

\section{REFERENCIAS BIBLIOGRÁFICAS}

1. Lane N. Osteoporosis: Metabolic Bone Disease. En: Firestein G, Budd R, Gabriel S. Kelley \& Firestein's. Textbook of Rheumatology. Tenth Edition. Vol. II. Philadelphia, PA: Elsevier; 2017. 17301750. ISBN: 978-0-323-31696-5.

2. Pinheiro $M B$, et al. Evidence of physical activity and osteoporosis prevention for people aged 65 years: a systematic review to inform the WHO guidelines on physical activity and sedentary behaiour. Int J Behav Nutr Phys Act [Internet]. 2020; 17(1): 1-53. Disponible en: https://doi.org/10.1186/ s12966-020-01040-4

3. Clark P, Chico G, Carlos F. Osteoporosis en América Latina: revisión de panel de expertos. Medwave [Internet]. 2013 [Consultado 18 enero 2021]; 13(8): e5791. Disponible en: Doi: 10.5867/ medwave.2013.08.5791

4. Riera-Espinoza G. Epidemiology of osteoporosis in Latin American 2008. Salud Pública de México [Internet]. 2009
[Consultado 22 de diciembre 2020]; 51(1): 52-55. Disponible en: http://www.redalyc. org/articulo.oa?id=10612571009

5. Clark P, Carlos F, Vázquez J. Epidemiology, costs and burden of osteoporosis in Mexico. Arch Osteoporos [Internet]. 2010 [Consultado 22 de diciembre 2020]; 5: 9-17. Disponible en: http://doi.org/10.1007/ s11657-010-0042-8

6. Contreras $M$, Bonilla $D$, Perez EE, et al. Niveles altos de IL-6 asociados a efectos sistémicos y locales en la artritis reumatoide. Medigraph [Internet]. 2015 [Consultado 18 de diciembre 2020]; 10(1): 38-42. Disponible en: https://www. medigraphic.com/pdfs/residente/rr-2015/ rr151f.pdf

7. Santillán S, Guerrero AP. Prevalencia de Osteoporosis en pacientes con Artritis Reumatoide [Trabajo de graduación]. Cuenca: Universidad del Azuay; 2015. p. 17. Disponible en: http://dspace.uazuay. edu.ec/handle/datos/5004

8. Schurman L, Galich A, González C, et al. Guías argentinas para el diagnóstico, la prevención y el tratamiento de la osteoporosis 2015. Medicina (Buenos Aires) [Internet]. 2017 [Consultado 17 diciembre 2020]; 77:46-60. Disponible en: http://www.osteoporosis.org.ar/pdf/ GuiasArgOP02017.pdf

9. Vargas F, Gallo F. A quién le pido una densitometría ósea. En: Guías de Actualización. Osteoporosis Manejo: prevención, diagnóstico y tratamiento. Sociedad Española de Medicina de Familia y Comunitaria, 2014. p. 21-25. Disponible en: https://www.semfyc.es 
10. Aizpurua I, Alvarez M, Echeto A, et al. Consenso sobre la osteoporosis postmenopáusica en la CAPV-Enero 2015. Osakidetza [Internet]. 2015 [Consultado el 15 de diciembre 2020]. Disponible en: https://www.euskadi.eus

11. Arnett FC, Edworthy SM, Bloch DA, et al. The American Rheumatism Association 1987 Revised Criteria For The Classification of Rheumatoid Arthritis. Arthritis and Rheumatism [Internet]. 1988 [Consultado 17 de diciembre de 2020]; 31: 315-24. Disponible en: DOI 10.1002/ art.1780310302

12. Aletaha FC, Neogi T, Silman AJ, et. al. 2010 Rheumatoid arthritis classification criteria: an American College of Rheumatology/ European League Against Rheumatism collaborative initiative. Arthritis Rheum [Internet]. 2010 [Consultado el 17 de diciembre de 2020]; 62(9): 2569-81. Disponible en: DOI 10.1002/art.27584.

13. Kanis JA, McCloskey $E$, Johansson $H$, et al. European guidance for the diagnosis and management of osteoporosis in postmenopausal women. Osteoporos Ing [Internet]. 2013 [Consultado el 18 de diciembre 2020]; 24(1): 23-57. Disponible en: DOI 10.1007/s00198-012-2074-y

14. Van Staat TP. The pathogenesis, epidemiology and management of gluclocorticoid-induced osteoporosis. Calif Tissue Int [Internet]. 2006 [Consultado el 19 de diciembre 2020]; 79: 129-137. Disponible en: DOI 10.1007/s00223-0060019-1

15. Adinoff AD, Hollister JR. Steroid-induced fractures and loss in patients with asthma. N Engl J Med [Internet]. 1983 [Consultado el 19 de diciembre de 2020]; 309: 265-268. Disponible en: DOI 10.1056/ NEJM198308043090502.
16. Clynes M, Jameson K, Prieto D, etal. Impacto de la artritis reumatoide y su tratamiento en caídas, fracturas y densidad mineral ósea en el biobanco del Reino Unido. Endocrinol frontal (Lausana) [Internet]. 2019 [Consultado 19 de diciembre de 2020]; 10: 187. Disponible en: DOI 10.3389/ fendo.2019.00817.eCollection2019

17. Guevara S, Feicán E, Peláez I, et al. Prevalencia de enfermedades reumáticas y calidad de vida en el pueblo indígena Saraguro, Ecuador: un estudio transversal de base comunitaria. J Clin Rheumatol [Internet]. 2020 [Consultado 19 de diciembre 2020]; 7(2): S139-S147. Disponible en: DOI 10.1097/RHU.0000000000001131,

18. Chelala C, Zaldívar A, Bruzón L. Factores de riesgo y la prevención de la osteoporosis. ccm Holguín [Internet]. 2017 [Consultado 28 de diciembre de 2020]; 21(4): 11741184. Disponible en: http://scielo.sld.cu/ scielo.php?script=sci_arttext\&pid=S156043812017000400017\&lng=es\&nrm=iso

19. Hernández A, Puerto I, Falcón A. Utilidad de la medición de la densidad mineral ósea en pacientes reumáticos. Rev Cuba Reumatol [Internet], 2014 [Consultado 28 de diciembre 2020];16(2):104-114. Disponible en: http://scielo.sld.cu/scielo. php?script $=$ sci_arttext $\&$ pid $=$ S181759962014000200006\&lng=es\&nrm=iso

20. Bullock J, Rizvi A, Ahmed J, et al. Rheumatoid Arthritis: a brief overview of the treatment. Med Princ Pract [Internet]. 2019 [Consultado el 28 de diciembre 2020]; 27(6): 501-507. Disponible en: DOI $10.1159 / 000493390$ 
21. Galarza D, Azpiri J, Colunga I, et al. Prevalencia de comorbilidades en pacientes mestizos mexicanos con artritis reumatoide. Rheumatol Int [Internet]. 2017 [Consultado el 28 de diciembre de 2020]; 37(9): 1507-1511. Disponible en: DOI 10.1007/s00296-017-3769-3

22. Fautrel B, Mitrovic S, Taylor P. Treatment of Rheumatoid Arthritis. En: Bijlsma J, Hachulla E. Eular Textbook on Rheumatic Diseases. Third Edition, 2018. p. 276-337. ISBN-10: 0727919245.

23. Ma CC, Xu SQ, Gong X, et al. Prevalence and risk factors associated with glucocorticoidinduced osteoporosis in Chinese patients with rheumatoid arthritis. Arch Osteoporos [Internet]. 2017 [Consultado el 28 de diciembre de 2020]; 12(1):33. Disponible en: DOI 10.1007/s11657-017-0329-0

24. Mikuls T, Saag K, Curtis J, et al. Prevalence of osteoporosis and osteopenia among African Americans with Early Rheumatoid Arthritis: The impact of ethnic-specific normative data. Journal of the National Medical Association [Internet]. 2005 [Consultado el 29 de diciembre de 2020];97(8):1155-1160. Disponible en: https://www.ncbi.nlm.nih.gov/pmc/ articles/PMC1364462/

25. Montañes JU. Artritis reumatoide y osteoporosis. Revisa Española de Enfermedades Metabólicas Óseas [Internet]. 2016 [Consultado el 29 de diciembre de 2020]; 9(2): 70-77. Disponible en: https://www.elsevier.es/es-revistareemo-70-estadisticas-10016983 